\title{
The Down-Regulation of SUZ12 Accelerates the Migration and Invasion of Liver Cancer Cells via Activating ERK1/2 Pathway
}

\author{
Cailin Xue ${ }^{1,2 *}$, Kunyuan Wang1*, Xiaofeng Jiang ${ }^{2}$, Chengxin Gu${ }^{1}$, Ganxiang Yu1 ${ }^{1}$, Yun Zhong ${ }^{3}$, Shiming \\ $\mathrm{Liu}^{3}$, Yuqiang Nie ${ }^{4}$, Yongjian Zhou ${ }^{4}$ and Hui Yang ${ }^{\bowtie}$ \\ 1. Department of Gastroenterology, The Second Affiliated Hospital of Guangzhou Medical University, Guangzhou, Guangdong 510260, P.R. China; \\ 2. Department of Hepatobiliary Surgery, The Second Affiliation Hospital of Guangzhou Medical University, Guangzhou, Guangdong 510260, P. R. China; \\ 3. Guangzhou Institute of Cardiovascular Disease, The Second Affiliated Hospital of Guangzhou Medical University, Guangzhou, Guangdong 510260, P.R. \\ China; \\ 4. Department of Gastroenterology, Guangzhou First People's Hospital of Guangzhou Medical University, Guangzhou, Guangdong 510180, P.R. China. \\ *Contributed equally
}

$\triangle$ Corresponding authors: Hui Yang, Department of Gastroenterology, The Second Affiliated Hospital of Guangzhou Medical University, 250 Changgang East Road, Guangzhou, Guangdong 510260, P.R. China, Tel: 86-020-34153522; Email: yanghui@gzhmu.edu.cn and Yongiian Zhou, Department of Gastroenterology, Guangzhou First People's Hospital of Guangzhou Medical University, Guangzhou, Guangdong 510180, P.R. China, Email: zhouyongiian1968@126.com

(c) Ivyspring International Publisher. This is an open access article distributed under the terms of the Creative Commons Attribution (CC BY-NC) license (https://creativecommons.org/licenses/by-nc/4.0/). See http://ivyspring.com/terms for full terms and conditions.

Received: 2018.09.14; Accepted: 2019.01.18; Published: 2019.02.23

\begin{abstract}
The suppressor of zest 12 (SUZ12), an essential subunit of the transcription polycomb repressive complex 2 (PRC2), has been found to be involved in HBV X-induced oncogenic transformation in hepatocellular carcinoma (HCC). However, the specific function of SUZ12 has not yet been determined in the pathogenesis of migration and invasion of HBV-associated HCC. Here, our results showed that SUZ12 was significantly down-regulated in HBV-related HCC tissues compared with adjacent non-tumor tissues by immunohistochemical and Western blot assays. The 5-years survival rate was worse in patients with low expression level of SUZ12. SUZ12 silencing increased the migration and invasion of HCC cells, and its overexpression impaired HCC cells migration and invasion. Knockdown of SUZ12 activated ERK1/2 pathway and increased MMP9 (matrix metallopeptidase 9) and MMP2 (matrix metallopeptidase 2) expression, whereas SUZ12 overexpression had opposite effects. Specific ERK1/2 inhibitor (SCH772984) significantly decreased $\mathrm{HCC}$ cells migration and invasion caused by SUZ12 shRNA. Thus, the liver cancer-down-regulated SUZ1 2 accelerated the invasion and metastasis of HCC cells. These effects might be associated with deregulation of SUZ12 activating ERK1/2, MMP2 and MMP9 in HCC cells.
\end{abstract}

Key words: SUZ12, Hepatocellular carcinoma, Migration, Invasion, ERK1/2

\section{Introduction}

Hepatocellular carcinoma (HCC) is the second leading cause of cancer-related death and the fifth most common cancer worldwide [1]. The aetiologies of HCC contain alcohol abuse, rare disorders such as a-1 antitrypsin deficiency and hemochromatosis [2], chronic infection with hepatitis B virus (HBV) and hepatitis $\mathrm{C}$ virus (HCV)[3-5], etc. Among them, persistent $\mathrm{HBV}$ infection remains a major factor in the pathogenesis of $\mathrm{HCC}[6]$. Despite the availability of
HBV vaccine and antiviral nucleoside analogs, some patients with long-term virological remission still develop liver cancer[7]. Therefore, identifying the risk factors for developing $\mathrm{HCC}$ in $\mathrm{HBV}$ carriers is important for the prevention of liver cancer.

Chronic liver inflammation and the effects of weakly oncogenic HBV $X$ protein are considered to be related the pathogenesis of HCC[5]. It has been reported that $\mathrm{HBx}$ acts as a cofactor in hepatocarcino- 
genesis by binding several nuclear proteins $[5,8]$. A genome-wide siRNA library screen performed in $\mathrm{HBx}$-expressing cells resulted in identification of nuclear proteins SUZ12 and ZNF198 (zinc finger, MYM-type 2), which is the downstream players in the HBx signaling network[9]. Moreover, HBx induces the PLK1 (Polo-like kinase 1)- and proteasomal-dependent degradation of SUZ12 and ZNF198, leading to the overexpression of SUZ12/PRC2 direct target genes [10].

SUZ12 is an essential component of the Polycomb Repressive chromatin remodeling Complex 2 (PRC2) that directs the (tri) methylation of lysine 27 on histone 3 (H3K27Me3) and gene silencing[11]. Recently, the effect of SUZ12 in cancers has drawn increasing attention. SUZ12 has been increasingly appreciated as a putative oncogene or tumour suppressor gene[12-15]. Some studies have reported that SUZ12 is frequently overexpressed in several solid cancers including head and neck squamous cell carcinoma (HNSCC), colorectal, ovarian and nonsmall lung cancer[16-18]. The reports showed that SUZ12 knockdown impaired the proliferation, invasion and metastasis of lung, bladder and gastric cancer cells $[13,18,19]$. The aberrant overexpression of SUZ12 was associated with aggressive clinicopathological characteristics and reduced overall and diseasefree survival of patients[12, 14, 20]. However, loss-offunction somatic alterations of SUZ12 contributed to the initiation and progression of malignant peripheral nerve sheath tumour[15]. Furthermore, SUZ12 was down-regulated in HBV-replicating cells and liver tumors of animals modeling $\mathrm{HBV}$-induced liver cancer[9, 10]. Previous study showed that SUZ12 knockdown led to defective DNA repair and p53 apoptosis, survival of HBV X protein (pX)-expressing cells from apoptosis induced by DNA damage, enhanced pX-induced polyploidy, and accelerated oncogenic transformation[9]. These findings indicate that absence of SUZ12 may be involved in the development and progression of HBV-mediated liver cancer. Nevertheless, the biological role of SUZ12 in HCC remains not well-understood.

The goals of this study are to examine whether SUZ12 acts as a suppressor gene in invasion and metastasis of HCC cells and further define the related signaling pathways in this disease model. We report that SUZ12 is down-regulated in HBV-related HCC tissues, and HCC patients with low SUZ12 expression level show a significantly poor prognosis as compared to patients with high SUZ12 expression. Moreover, our data provides direct evidence that the liver cancer-down-regulated SUZ12 accelerates the invasion and metastasis of HCC cells. These effects may be associated with deregulation of SUZ12 activating ERK1/2, MMP2 and MMP9 in HCC cells.

\section{Materials and Methods}

\section{Tissue microarray and samples}

A tissue microarray with 95 paired of liver cancer tissues and the corresponding non-tumor tissues was purchased from Outdo Biotech Company (diameter, $1.5 \mathrm{~mm}$, HLiv-HCC180Sur-05; Shanghai, China). 18 pairs of fresh HCC tissues and adjacent non-tumor tissues were collected from patients who initially underwent surgery and were histologically diagnosed with HCC between January 2015 and December 2016 at the Second Affiliated Hospital of Guangzhou Medical University (Guangzhou, China). Ethical approval for the study was given by the Research Ethics Committee of the Second Affiliated Hospital of Guangzhou Medical University. All eligible patients provided informed consent prior to the collection of HCC tissues and corresponding adjacent non-tumor tissues. Each sample was frozen at $-80^{\circ} \mathrm{C}$ prior to protein extraction.

\section{Cell lines}

The human HCC cell lines SMMC-7721 was obtained from Library of Tumor Cells of Chinese Academy of Medical Sciences (Shanghai, China) and Hep3B from the American Type Culture Collection (ATCC, USA), which were cultured at $37^{\circ} \mathrm{C}$ in a humidified atmosphere containing $5 \% \quad \mathrm{CO}_{2}$ in Dulbecco's modified Eagle's medium (Invitrogen, Carlsbad, CA, USA) supplemented with $10 \%$ fetal bovine serum (Invitrogen), 100U/ml penicillin and $100 \mu \mathrm{g} / \mathrm{ml}$ streptomycin (Invitrogen).

\section{Cells transfection}

The sh-SUZ12 lentiviral vector was obtained from Santa Cruz Biotechnology (Santa Cruz, CA, USA) and was used to knock down the SUZ12 expression. The SUZ12 shRNA sequence was as follows: GATCCGAATCTCATAGCACCAATATTCAAGAG ATATTGGTGCTATGAGATTCTTTTT. The negative lentiviral vector containing non-silencing short hairpin RNA was used as a control. The SUZ12 overexpressed lentiviral vector and the control vector were obtained from Fulengen Company (Guangzhou, China). SMMC-7721 and Hep3B cells were infected with either of the lentiviral vectors encoding sh-SUZ12 or SUZ12 overexpressed sequences and the corresponding negative control vectors. Vectors were transfected using Lipofectamine ${ }^{\circledR}$ LTX and PLUS ${ }^{\mathrm{TM}}$ reagents or Lipofectamine ${ }^{\circledR}$ RNAiMAX reagent (Invitrogen, Carlsbad, CA, USA) according to the instructions of manufacturer. The efficiencies of RNA interference or overexpression were determined by Western blot assay. 


\section{Western blot analysis}

The extraction of total protein lysate and SDS-PAGE were performed according to previously described [21]. The primary antibodies included antiSUZ12, anti-E-cadherin, anti-N-cadherin, anti-Vimentin, anti-MMP-9, anti-MMP-2, anti-P-ERK1/2 and anti-GAPDH, which were purchased from Cell Signaling Technology (Danvers, MA).

\section{Immunohistochemical assay}

Immunohistochemical staining was performed by using a Dako Envision System (Dako, Carpinteria, CA, USA) following the manufacturer's recommended protocol. The tissue sections were treated with $3 \%$ hydrogen peroxide to inactivate the endogenous peroxidase, and antigens were retrieved using a citrate buffer. After incubating in PBS containing 5\% normal goat serum (cat. no. C-0005; BIOSS, Beijing, China) at room temperature for $1 \mathrm{~h}$, the sections was incubated with the anti-SUZ12 antibody at $4^{\circ} \mathrm{C}$ overnight (ab236322; Abcam, Cambridge, UK). Then, the sections were rinsed with PBS and incubated with a secondary antibody (Dako; Agilent Technologies, Inc., Santa Clara, CA, USA) at room temperature for 1 h. Finally, 3,3'-diami-nobenzidine was used to induce a color reaction. IHC results were assessed under a light microscope at $\times 200$ magnification.

Tissue sections stained with IHC were scored by two pathologists blinded to the clinical parameters, respectively. According to the percentages of positive staining areas in tumor tissue or normal tissue, the staining intensity was scored as 0 (negative), 1 (weak), 2 (medium) and 3 (strong), and the extent of staining was scored as $0(0 \%), 1(1-25 \%), 2(26-50 \%), 3$ $(51-75 \%)$ and $4(76-100 \%)$. The sum of the intensity and extent scores was used as the final staining score (0-7) for SUZ12. The score $<4$ was divided into low expression group, and $\geq 4$ divided into high expression group.

\section{Cell viability and proliferation assays}

The HCC cells transfected with sh-SUZ12 or LV-SUZ12 and the corresponding lentiviral vectors were seeded in 96-well plates at $5 \times 10^{3}$ cells per well. The protocol of CellTiter-96 Aqueous-One Solution Cell Proliferation (MTT) Assay kit (Promega, Madison, WI, USA) was described previously[22]. The proliferation ability of transfected cells was detected by the EdU incorporation assays using EdU Cell Proliferation Assay Kit (Ribobio, Wuhan, China) and colony formation assays. After incubation with $50 \mu \mathrm{M}$ EdU, cells were fixed and stained with EdU according to the manufacturer's instructions and recommenddations. For colony formation assay, cells were seeded at a density of 2000 cells in one $6 \mathrm{~cm}$ culture dish and cultured for 2 weeks, and then colonies were stained with $1 \%$ crystal violet and counted.

\section{Transwell migration and Boyden chamber invasion assays}

The SMMC-7721 and Hep3B cells were transfected with sh-SUZ12, LV-SUZ12 and the negative control lentiviral vectors for $24 \mathrm{~h}$. Chambers (Corning, USA) with or without matrigel (BD Biosciences, San Jose, CA, USA) bedding were placed in 24-well plates. For transwell migration assay, $5 \times 10^{4}$ cells suspended in $200 \mu \mathrm{l}$ serum-free DMEM media were seeded into the upper chamber of Transwell. $600 \mu 1$ DMEM containing 10\% FBS was added into the bottom chamber. After incubation at $37^{\circ} \mathrm{C}$ for $24 \mathrm{~h}$, cells adhered on the lower surface of filters were washed twice with PBS and fixed with 100\% methanol at room temperature for 30 minutes with $4 \mathrm{~g} / \mathrm{L}$ crystal violet. Staining results were observed and imaged under a light microscope (Olympus BX 51; Olympus Corporation, Tokyo, Japan). Cell numbers in 5 random fields in each replicate were counted, and each assay was performed for three times. For Boyden chamber invasion assay, the procedure was the same with transwell migration assay except that the transwell membrane was precoated with matrigel.

\section{Statistical analysis}

Statistical analyses were conducted using SPSS version 17.0 software (SPSS, Inc., Chicago, IL, USA). The quantitative data was shown as means \pm SD from three independent experiments. Differential expression levels of SUZ12 in HCC tissues and matched adjacent tissues detected by IHC assay were analyzed by a non-parametric Wilcoxon method. Student's t-test was used to analyze the significance of data from colony formation assay, Western blot assay and Transwell migration and Boyden chamber invasion assays. Multiway classification analysis of variance test was performed for results from MTT assays. Survival curves were calculated using Kaplan-Meier and log-rank tests. A value of $P<0.05$ indicated a significant difference.

\section{Results}

\section{The expression levels of SUZ12 protein is down-regulated in HCC tissues}

To investigate the potential role of SUZ12 in HCC, we analyzed the protein expression level of SUZ12 on a tissue microarray of HCC by IHC. As shown in Figure 1A, the positive immunostaining of SUZ12 protein was mainly located in the cytoplasm of non-tumor tissues, which appeared as granular brown-colored staining. SUZ12 protein was lowly expressed in the majority of tumor tissues as 
compared with that in the corresponding non-tumor tissues ( $P=0.044$; Fig.1B). Kaplan-Meier and log-rank test analyses revealed that patients with highly expressed SUZ12 in tumor tissues had a significantly longer survival time than those with low SUZ12 expression levels ( $P=0.004$; Fig. $1 C)$. These findings indicate that SUZ12 protein is down-regulated in HCC tissues, and its expression is positively correlated with the overall survival of patients with HCC.

\section{SUZ1 2 exerts its biological effects through regulating the migration and invasion of $\mathrm{HCC}$ cells, but not the proliferation}

To explore the biological function of SUZ12 in HCC development and progression, Hep3B and SMMC-7721 cells were selected for SUZ12 knockdown and overexpression. The shRNA-SUZ12 could effectively silenced the protein expression of SUZ12, and the LV-SUZ12 increased SUZ12 protein expression (Fig.2A). To investigate whether endogenous silence or overexpression of SUZ12 affected the proliferative capacity of HCC cells, EdU and MTT assays were performed. The results showed that SUZ12 knockdown or overexpression had no obvious effect on the proliferation of Hep3B and SMMC-7721 cells (Fig.2B-C). Meanwhile, we observed the similar

A

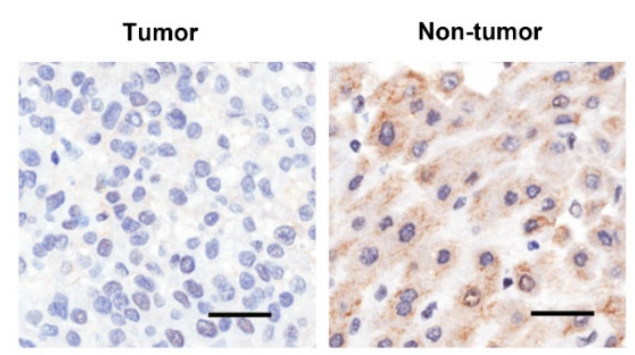

B

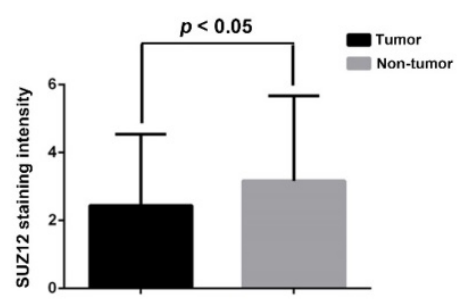

C

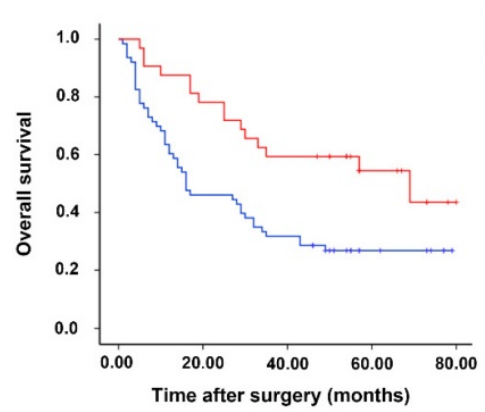

Figure 1. SUZ12 protein expression is decreased in HCC specimens. (A) Immunohistochemical staining of SUZ1 2 protein on HCC tissues and the corresponding non-tumor tissues. Representative images with original magnification at $\times 200$ are shown, scale bars $=100 \mu \mathrm{m}$. (B) The expression of SUZ12 protein in HCC tissues (2.442 \pm 2.100 ) was lower than that in non-tumor tissues (3.169 \pm 2.510$)$. (C) Kaplan-Meier and log-rank test analyses of overall survival in $95 \mathrm{HCC}$ patients based on SUZ12 expression level of HCC tissues. phenomenon in colony formation assay that there was no significant difference in the number of colonies between the control and knockdown or overexpressed groups (Fig.2D).

Next, we examined the effect of SUZ12 on the migration and invasion of HCC cells. The migration and Matrigel invasion assays showed that Hep3B cells transfected with sh-SUZ12 exhibited strong migratory and invasive capabilities. Conversely, the migration and invasion ability were impaired in Hep3B cells transfected with LV-SUZ12 (Fig.3A-B, P<0.001). Similar results were found in SMMC-7721 cells that transfected with shSUZ12 or LV-SUZ12 (Fig.3C-D, $P<0.001)$. Together, SUZ12 functions as a tumor suppressor gene by restricting the migration and invasion of HCC cells.

\section{Extracellular signal-regulated kinase $1 / 2$ pathway is involved in regulating the biological function of SUZ12 in HCC}

Epithelial-mesenchymal transition (EMT) is thought to be a key process that contributes to tumor cells migration and invasion[23-25]. Compared with the corresponding control cells, Western blot analysis showed no significant changes in the expression of epithelial markers E-cadherin and mesenchymal markers N-cadherin and vimentin in SUZ12 knockdown or overexpressed HCC cells (Fig.4A). Previous reports have suggested that Matrix metalloproteinase 9 (MMP9) and MMP2 play an important role in cancer cells migration and invasion [26, 27]. Thus, we evaluated whether SUZ12 affected the expression of MMP9 and MMP2 by Western blot assay. As shown in Figure 4A, the expression of MMP9 and MMP2 were increased when SUZ12 was knocked down in Hep3B and SMMC-7721 cells, whereas MMP9 and MMP2 expression levels decreased in SUZ12 overexpressed cells. In addition, it was reported that ERK pathway regulated the metastasis and invasion of HCC[28, 29]. We found that the expression of phosphorylated ERK1/2 was similar with MMP9 and MMP2, which was highly expressed in SUZ12 knockdown cells and lowly expressed in SUZ12 overexpressed HCC cells (Fig.4A). The gray scale ratios of SUZ12, E-cadherin, N-cadherin, vimentin, MMP9, MMP2 and PERK1/2 to GAPDH were displayed 
in Figure 4B. These results indicate that increased MMP9, MMP2 and P-ERK1/2 protein expression contribute to the migration and invasion of HCC.

To further confirm that ERK signaling pathway was involved in the function of SUZ12 in HCC, the ERK activation/phosphorylation inhibitor SCH7729 84 was used to treat Hep3B and SMMC-7721 cells[30]. We observed that the migration and invasion abilities of SUZ12-silenced HCC cells were significantly inhibited after SCH772984 treatment (Fig.5A-B). Meanwhile, the expression levels of P-ERK1/2, MMP9 and MMP2 proteins were restored when treated with SCH772984 in sh-SUZ12 HCC cells (Fig.5C-D). Collectively, SUZ12 suppresses the migration and invasion of HCC cells by modulating ERK inactivation.

A

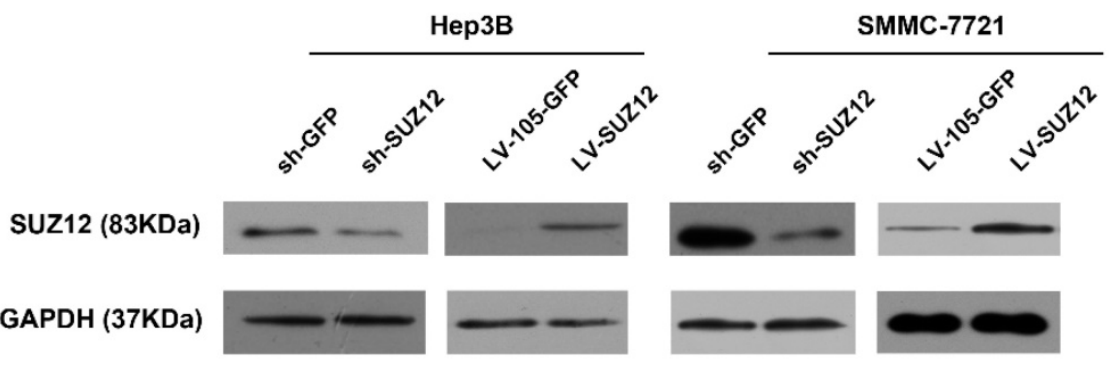

B
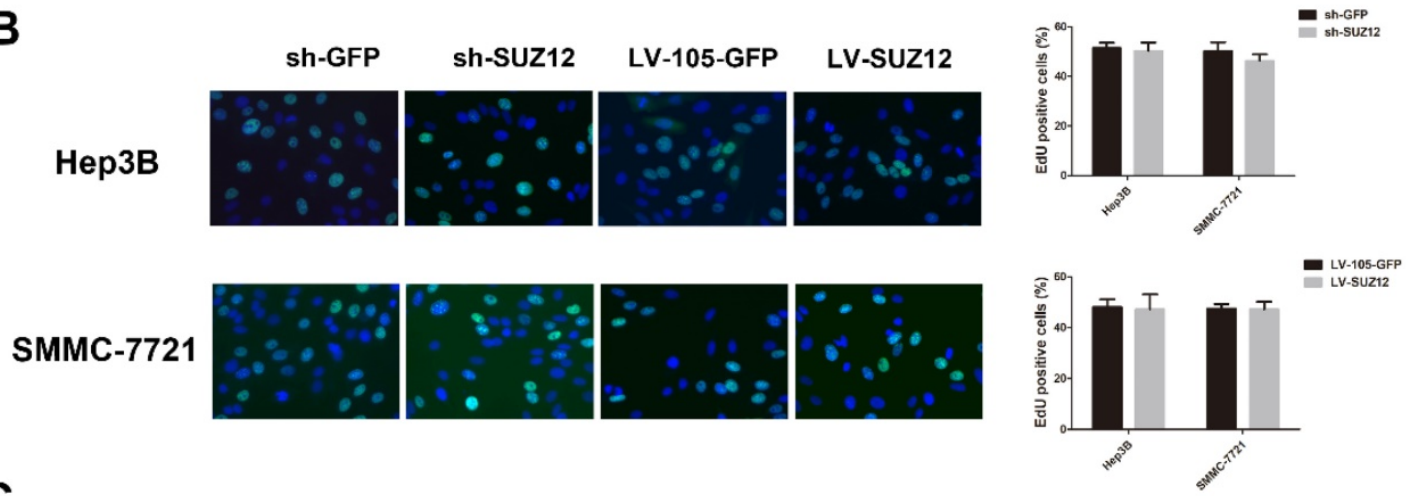

C
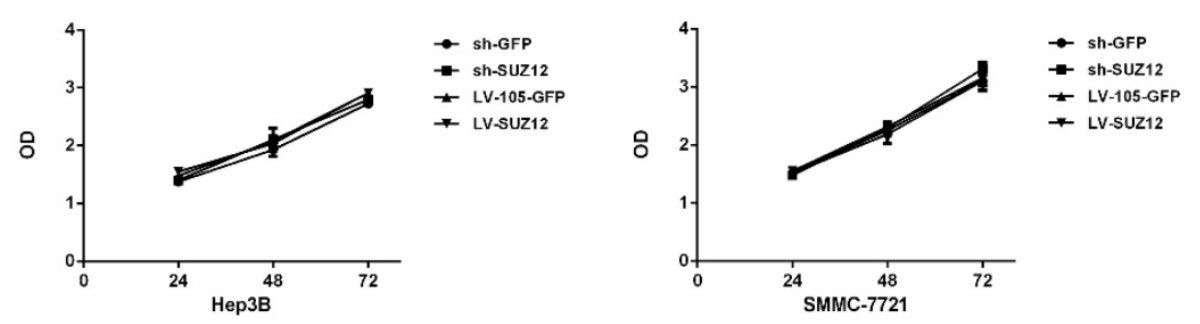

D

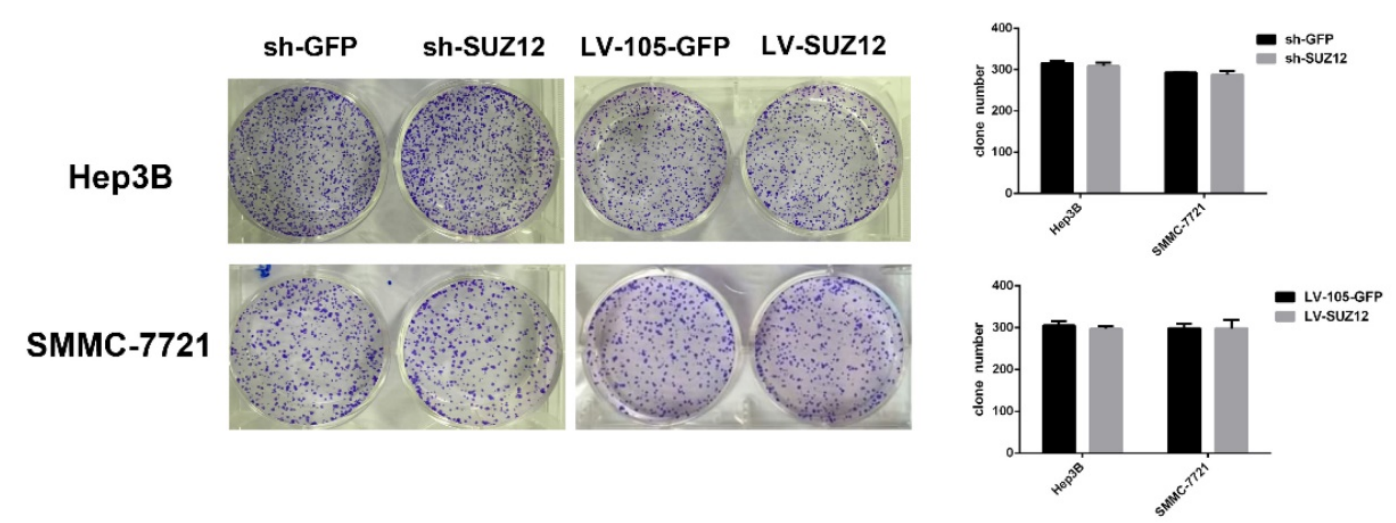

Figure 2. SUZ1 2 knockdown or overexpression does not affect the proliferation of HCC cells. (A) Western blot analysis showed the expression levels of SUZ12 protein in Hep3B and SMMC-7721 cells transfected with sh-SUZ12 or LV-SUZ12. GAPDH served as a loading control. (B-D) The proliferative capacities of Hep3B and SMMC-7721 cells with SUZ12 knockdown or overexpression, as determined by EdU, MTT and colony formation assays (magnification: B, $\times 400$ ). 
A

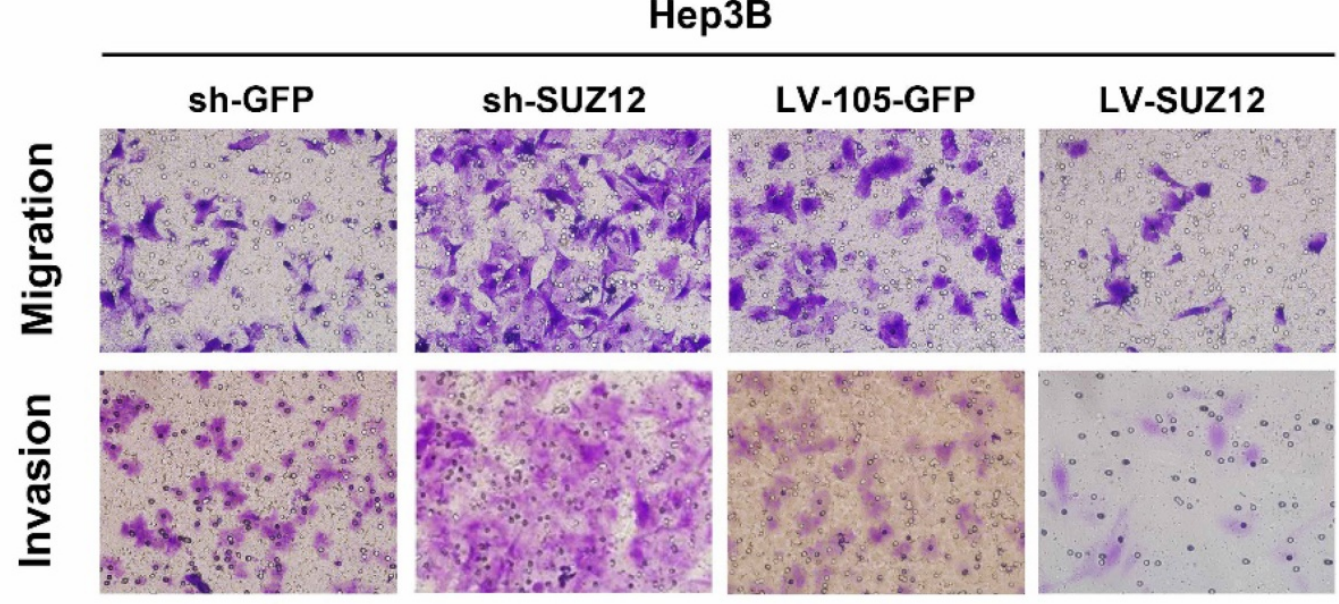

B
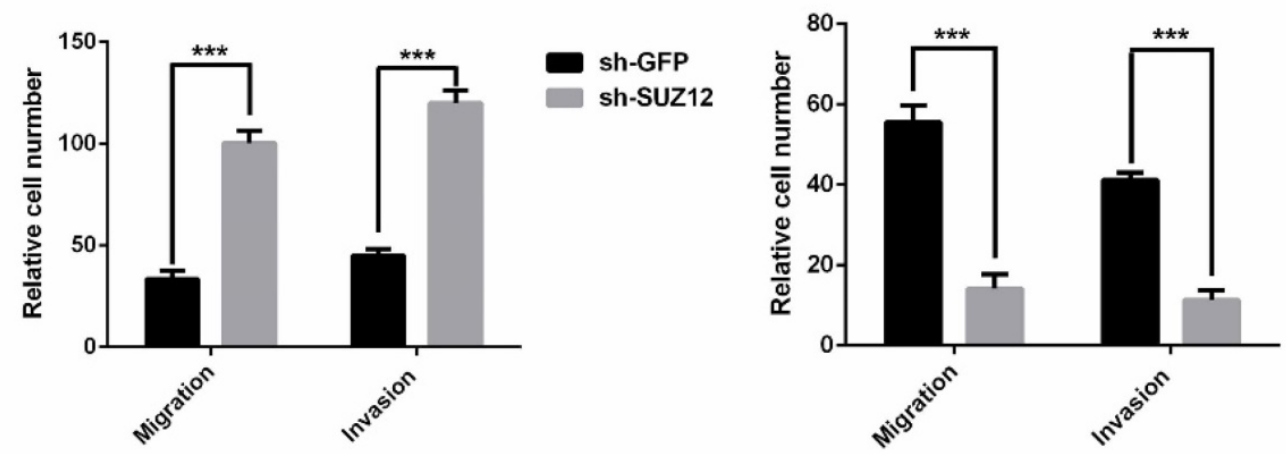

LV-GFP

LV-SUZ12

C

SMMC-7721

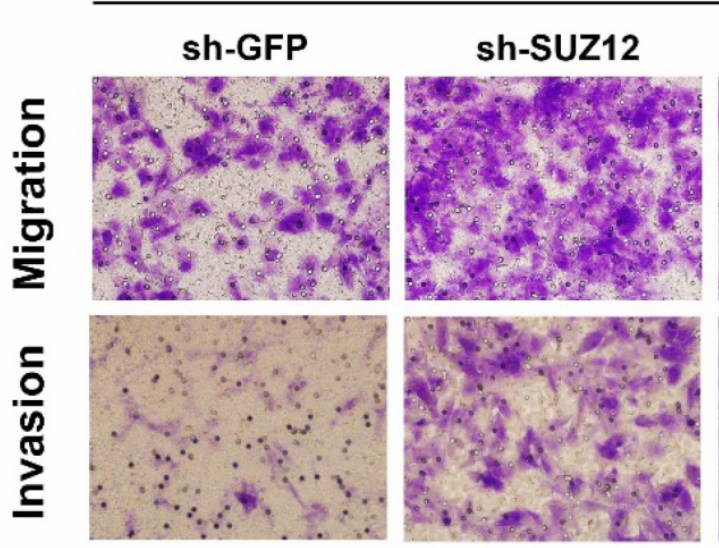

\section{LV-105-GFP}

LV-SUZ12

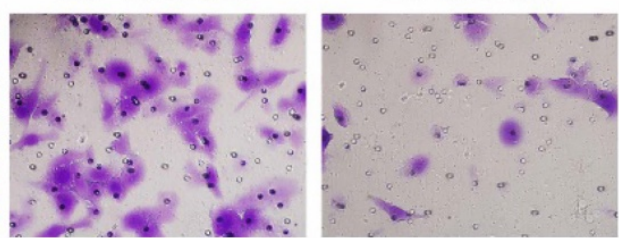

D
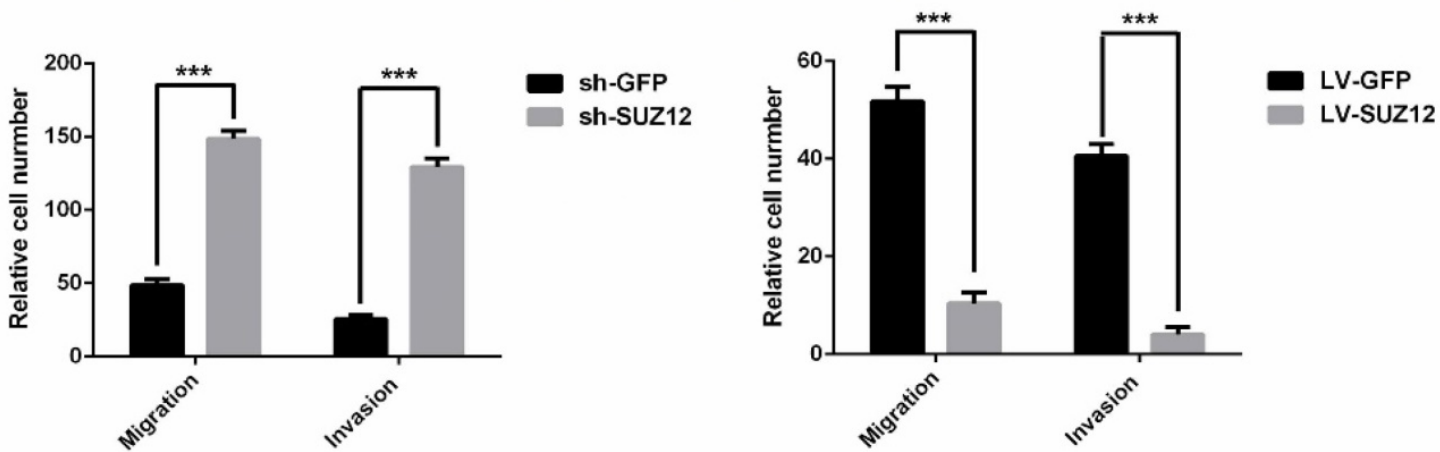

Figure 3. SUZ12 regulates the migration and invasion abilities of HCC cells. (A-C) Representative images of Transwell migration assay and Boyden chamber invasion assay in SUZ12-silenced or overexpressed Hep3B and SMMC-7721 cells (magnification: $\times 400$ ). (B-D) The bar charts represented the average number of migratory and invasive cells from five random microscopic fields. The data represent the Mean $\pm S D, * * * P<0.001$. 
A

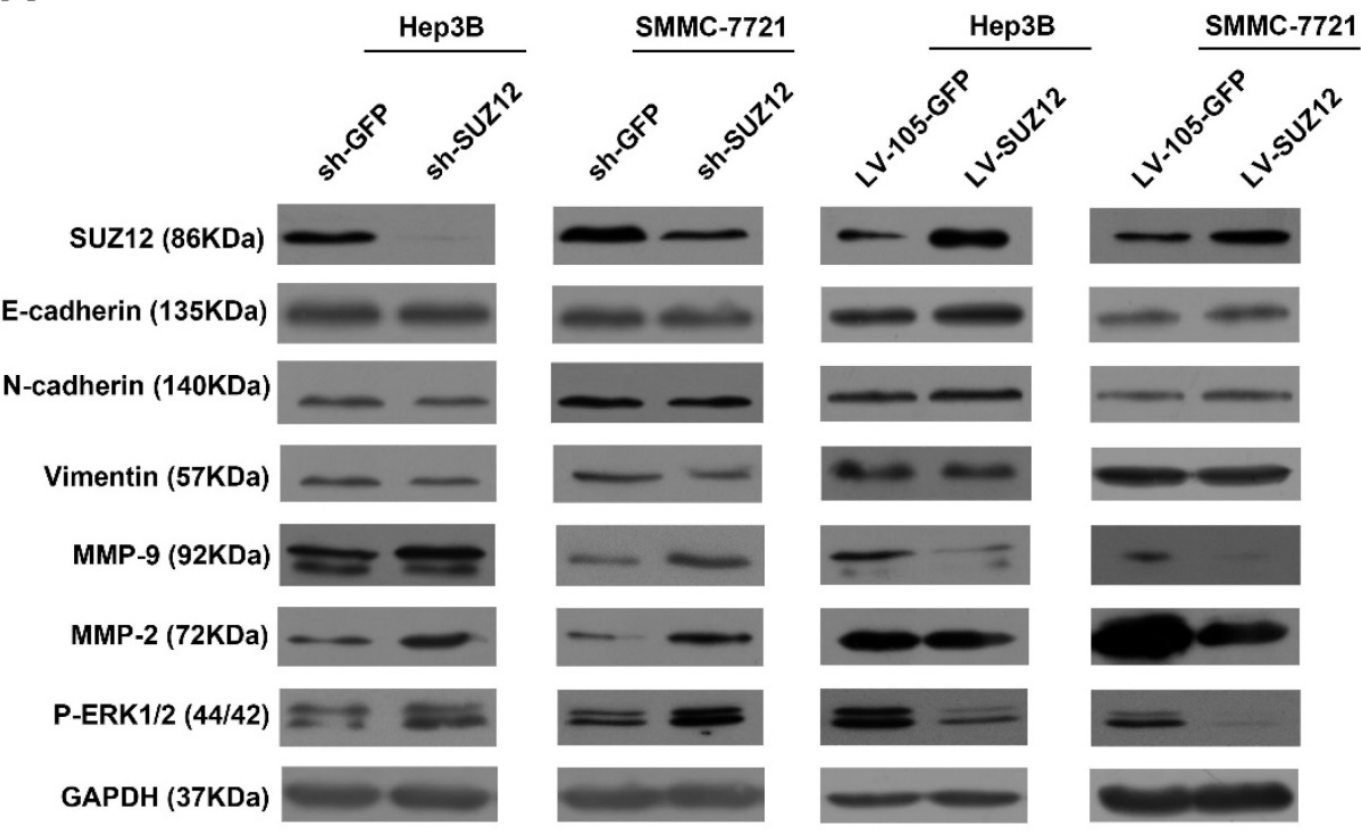

B
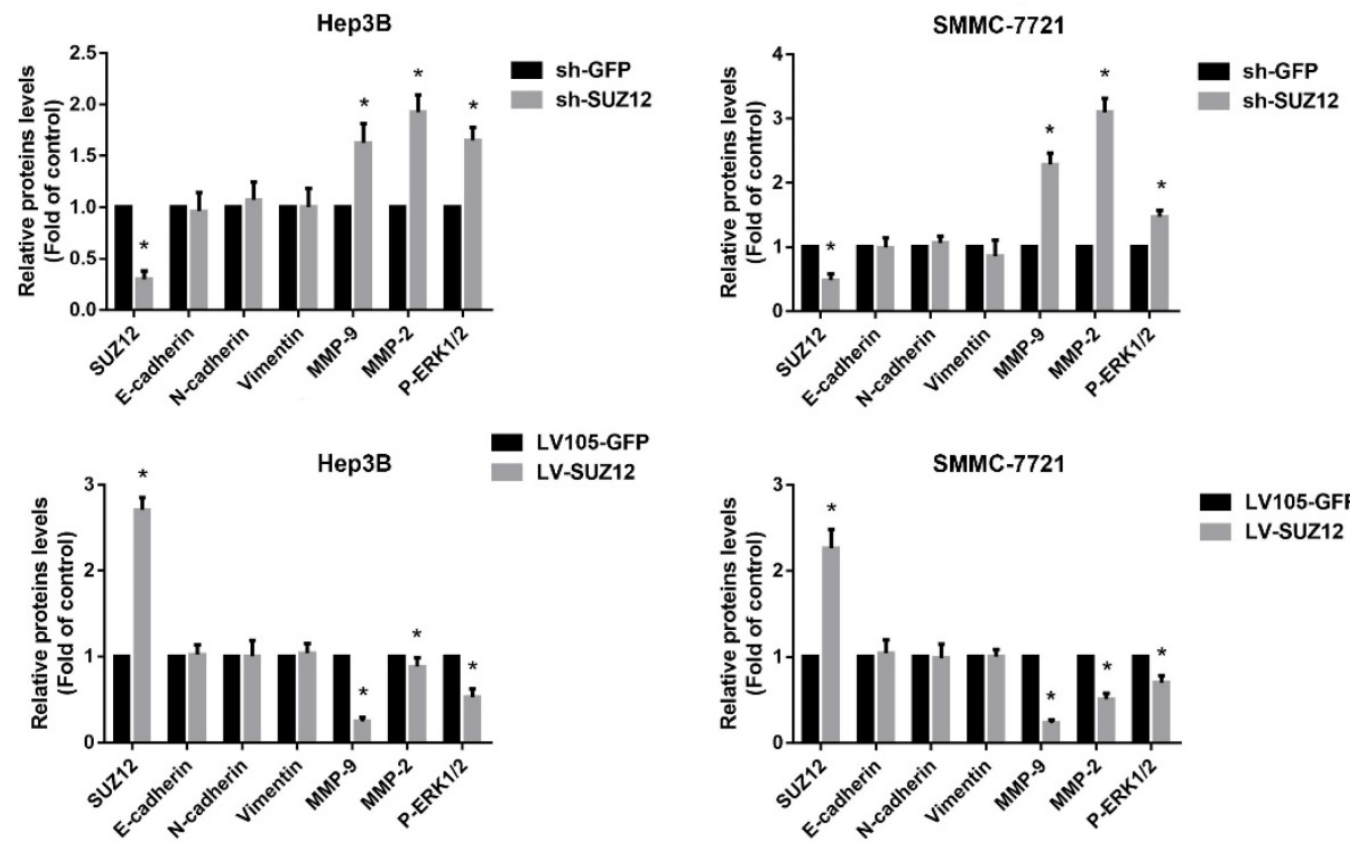

Figure 4. The expression levels of migration and invasion-associated molecules in SUZ12-silenced or overexpressed HCC cells. (A) The expression levels of SUZ12, E-cadherin, N-cadherin, vimentin, MMP9, MMP2 and P-ERK1/2 proteins were determined by Western blot assay in SUZ12-silenced or overexpressed Hep3B and SMMC-7721 cells. (B) Comparisons of the expressions of E-cadherin, N-cadherin, Vimentin, MMP9, MMP2 and P-ERK1/2 proteins in SUZ12-silenced and overexpressed HCC cells, as well as the corresponding control cells. The data represent the Mean \pm SD, $* P<0.05$.

The protein expression level of SUZ12 is negatively correlated with P-ERK1/2 protein level in HCC tissues

To investigate the correlation between the expression of SUZ12 and P-ERK1/2 protein in HCC tissues, Western blot assay was used to detect their expression levels. The results showed that SUZ12 protein expression was down-regulated in 11 of the
HCC tissues, while P-ERK1/2 protein expression was up-regulated in the same tissues. Similarly, we found that the protein expression of SUZ12 increased in the corresponding normal liver tissues while P-ERK1/2 decreased (Fig.6). Taken together, these results further confirm that the expression level of SUZ12 is down-regulated in HCC tissues and negatively correlate with the expression level of P-ERK1/2. 
A
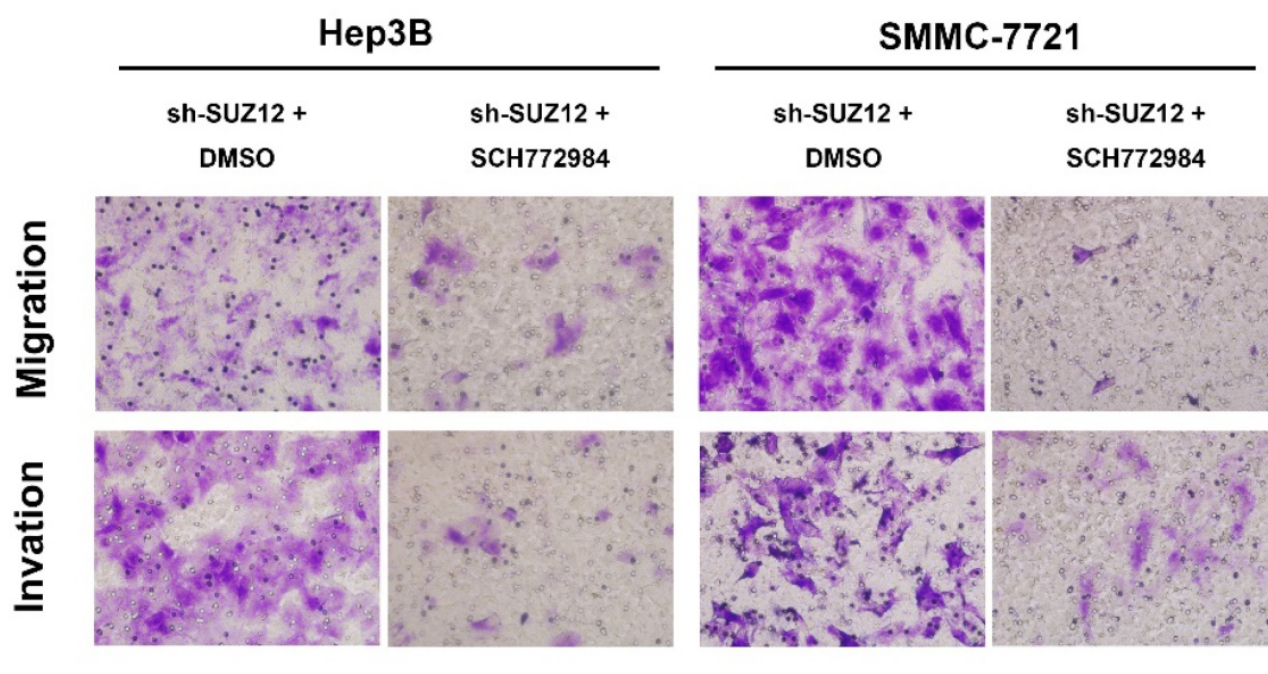

B

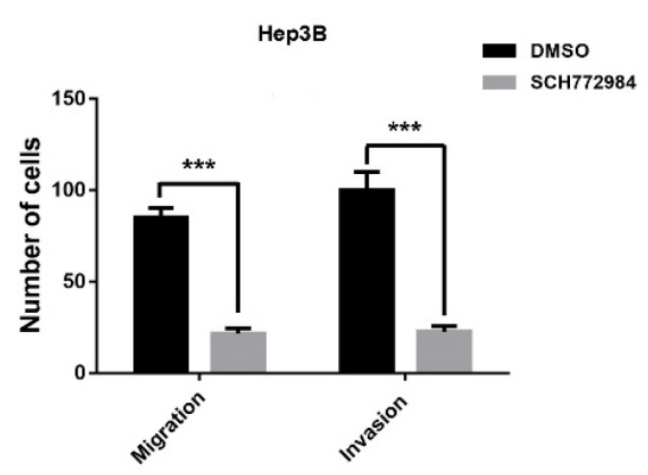

C
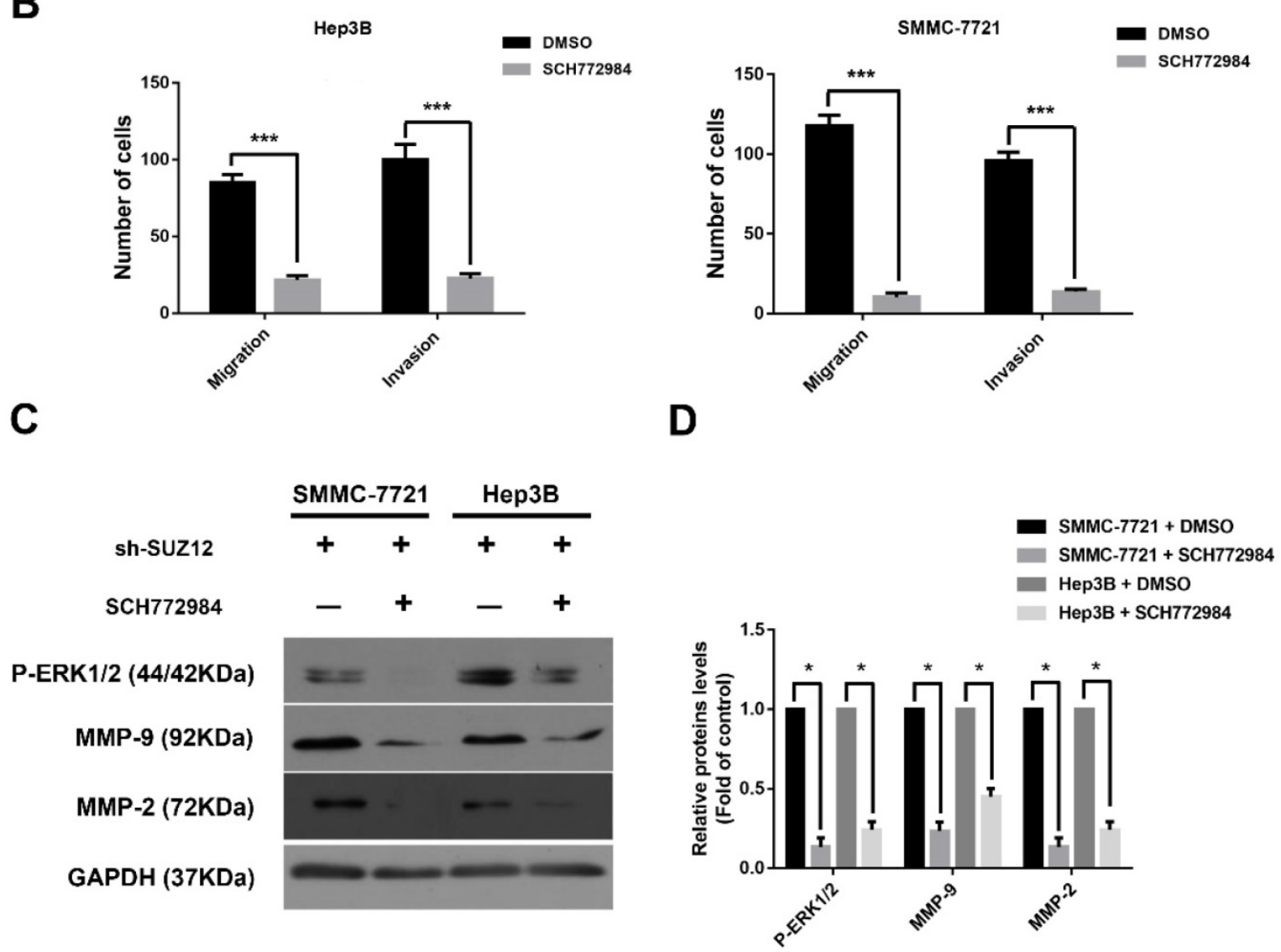

Figure 5. SUZ12 inhibits the migration and invasion of HCC cells via inactivating the ERK1/2 signaling pathway. (A) Representative images of Transwell migration and invasion assays in SUZ12-silenced Hep3B and SMMC-7721 cells treated with SCH772984 or control (magnification: $\times 400)$. (B) The bar charts stood for the average number of migratory and invaded cells from five random microscopic fields. (C) Western blot analysis of P-ERK1/2, MMP9 and MMP2 proteins in SUZ12-silenced Hep3B and SMMC-7721 cells treated with SCH772984 or control. (D) The levels of the indicated proteins were quantified with gray value. The data represent the Mean $\pm S D, * * * P<0.001, * P<0.05$.

\section{Discussions}

The present study demonstrated that SUZ12 was down-regulated in HBV-related HCC specimens, and its expression was positively correlated with the OS of HCC patients. SUZ12 acted as a tumor suppressor by inhibiting the migration and invasion of HCC, but not cell proliferation. Meanwhile, we found that the P-ERK1/2 pathway is involved in regulating the biological function of SUZ12 in HCC.

Previous studies have revealed that SUZ12 is an oncogene that promotes tumorigenesis and a 
potential biomarker as well as anticancer therapeutic target in various tumors[12, 14, 19, 20]. Wu Y et al.[12] reported that SUZ12 was overexpressed in HNSCC, which elevated SUZ12 expression significantly associated with cervical node metastasis and the reduced overall survival. In epithelial ovarian cancer (EOC), SUZ12 was up-regulated in EOC tissues, and SUZ12 overexpression predicted shorter overall survival[17]. However, our study showed that the expression level of SUZ12 protein was decreased in HBV-related HCC tissues compared with the corresponding para-tumor and non-tumor tissues (Fig.1B and Fig.6). This is consistent with previous findings that SUZ12 is down-regulated in HBV-replicating cells and liver tumor tissues of HBV-induced HCC animal models[9, 10], which down-regulation of SUZ12 occurs in liver tumor of woodchucks chronically infected by woodchuck hepatitis virus[31]. Moreover, the analysis performed in our study showed a significant correlation between SUZ12 overexpression and better clinical outcome (Fig.1C). These current researches strongly suggest the tumor suppressing role of SUZ12 in HCC.

Accumulating evidence has indicated that SUZ12 exerts oncogenic role by promoting the proliferation, migration and invasion of tumor cells in various malignancies[12, 13, 18, 19, 32]. Nevertheless, Lee $\mathrm{W}$ et al. reported that SUZ12 was a tumor suppressor in malignant peripheral nerve sheath tumors, which overexpression of SUZ12 reduced the proliferation of SUZ12-deficient cells[15]. In HBVmediated HCC pathogenesis and HBV replication,
Wang W and Studach LL et al. found that knockdown of SUZ12 increased cell survival and proliferation in conditions of HBV X-mediated apoptosis and HBV $\mathrm{X}$-induced polyploidy and oncogenic transformation $[9,10,33,34]$. Therefore, we explored the biological role of SUZ12 in HCC through detecting the proliferation, migration and invasion of hepatoma cells. However, our study showed that SUZ12 did not affect the proliferatory ability of HCC cells (Fig.2). We found that knockdown of SUZ12 facilitated HCC cells migration and invasion by inducing MMP-9 and MMP-2 expression, and vice versa (Fig.3 and Fig.4). Although most studies have supported the concept that EMT promotes cancer metastasis[25], which endows cancer cells with increased migratory and invasive behavior[35], the expression levels of the epithelial and mesenchymal proteins such as E-cadherin, $\mathrm{N}$-cadherin and Vimentin remain unchanged in SUZ12 knockdown or overexpressed HCC cells (Fig.4). It has been well known that SUZ12 is an essential component for activity of the polycomb repressive complex PRC2 that regulates the expression of many developmental and signaling genes[36]. Consequently, we speculated that MMP-9 and MMP-2 may be the target genes of SUZ12. These results demonstrate that SUZ12 serves as a tumor suppressor in HCC by restricting the migration and invasion of liver cancer cells.

The ERK1/2 signalling pathway is deregulated in many kinds of cancers and promotes cancer development and progression[37]. Guo $\mathrm{Y}$ et al. reported that Homeobox D10 suppressed HCC growth by inhibiting ERK signaling[38]. In FGFR1-amplified lung cancer, activation of FGFR1 promotes cell proliferation, EMT and metastasis by regulating FGFR1-ERK1/2-SOX2 axis [39]. In HER $2+$ breast cancer, Notch-1-PTEN-ERK1/2 signaling axis promotes cell proliferation and stem cell survival[40]. Here, we found that SUZ12 suppressed the migratory and invasive capabilities of HCC cells by inhibiting ERK1/2 signaling. And SCH772984, an inhibitor of ERK1/2 catalytic activity, could attenuates the migration and invasion of hepatoma cells induced by SUZ12 silencing (Fig.4 and Fig.5). In addition, our results showed that there was a negative correlation between the protein expression of SUZ12 and ERK1/2 in HBV-related HCC tissues (Fig.6). Current research has demonstrated the potential mechanism by which down-regulation of SUZ12 promotes HCC progression.
Figure 6. The correlation of SUZ12 and P-ERK1/2 protein expression in HCC tissues. Western blot assay was performed to detect the protein expression levels of SUZ12 and P-ERK $1 / 2$ in $\mathrm{HCC}$ tissues, adjacent non-tumor tissues and corresponding normal liver tissues of 18 patients with HCC. T stands for tumor tissues; AT stands for adjacent tissues of HCC tissues; $\mathrm{N}$ stands for normal liver tissues. 
Nevertheless, further studies are needed to elucidate the exact mechanism of SUZ12 in HCC.

In summary, our findings provide experimental evidence that SUZ12 acts as a tumor suppressor to inhibit the migration and invasion of HCC cells by decreasing activation of the ERK1/2 pathway. Thus, SUZ12 can be considered as a potential prognostic indicator and therapeutic target in HCC patients.

\section{Acknowledgments}

This study was supported by grants from the Science and Technology Program of Guangzhou (no. 201707010470), and the National Natural Science Foundation of China (nos. 81372634 and 81600350), the Guangdong Natural Science Funds for Distinguished Young Scholar (no. S2013050014121) and a research project from Guangdong Province Office of Education (no. 2015KTSCX117).

\section{Competing Interests}

The authors have declared that no competing interest exists.

\section{References}

1. Ferlay J, Soerjomataram I, Dikshit R, Eser S, Mathers C, Rebelo M, et al. Cancer incidence and mortality worldwide: sources, methods and major patterns in GLOBOCAN 2012. Int J Cancer 2015;136: E359-86.

2. Llovet JM, Zucman-Rossi J, Pikarsky E, Sangro B, Schwartz M, Sherman M, et al. Hepatocellular carcinoma. Nat Rev Dis Primers 2016;2: 16018

3. Liu J, Yang HI, Lee MH, Lu SN, Jen CL, Batrla-Utermann R, et al. Spontaneous seroclearance of hepatitis B seromarkers and subsequent risk of hepatocellular carcinoma. Gut 2014;63: 1648-57.

4. Torre LA, Bray F, Siegel RL, Ferlay J, Lortet-Tieulent J, Jemal A. Global cancer statistics, 2012. CA Cancer J Clin 2015;65: 87-108.

5. Levrero M, Zucman-Rossi J. Mechanisms of HBV-induced hepatocellular carcinoma. J Hepatol 2016;64: S84-101.

6. Lin $\mathrm{CL}, \mathrm{Kao} \mathrm{JH}$. Review article: the prevention of hepatitis B-related hepatocellular carcinoma. Aliment Pharmacol Ther 2018.

7. Yu MW, Lin CL, Liu CJ, Yang SH, Tseng YL, Wu CF. Influence of Metabolic Risk Factors on Risk of Hepatocellular Carcinoma and Liver-Related Death in Men With Chronic Hepatitis B: A Large Cohort Study. Gastroenterology 2017;153: 1006-17.

8. Cougot D, Wu Y, Cairo S, Caramel J, Renard CA, Levy L, et al. The hepatitis B virus $X$ protein functionally interacts with CREB-binding protein/p300 in the regulation of CREB-mediated transcription. J Biol Chem 2007;282: 4277-87.

9. Wang W, Studach LL, Andrisani OM. Proteins ZNF198 and SUZ12 are down-regulated in hepatitis B virus (HBV) X protein-mediated hepatocyte transformation and in HBV replication. Hepatology 2011;53: 1137-47.

10. Studach LL, Menne S, Cairo S, Buendia MA, Hullinger RL, Lefrancois L, et al. Subset of Suz12/PRC2 target genes is activated during hepatitis B virus replication and liver carcinogenesis associated with $\mathrm{HBV} X$ protein. Hepatology 2012;56: 1240-51.

11. Margueron R, Reinberg D. The Polycomb complex PRC2 and its mark in life. Nature 2011;469: 343-9.

12. Wu Y, Hu H, Zhang W, Li Z, Diao P, Wang D, et al. SUZ12 is a novel putative oncogene promoting tumorigenesis in head and neck squamous cell carcinoma. J Cell Mol Med 2018.

13. Lee SR, Roh YG, Kim SK, Lee JS, Seol SY, Lee HH, et al. Activation of EZH2 and SUZ12 Regulated by E2F1 Predicts the Disease Progression and Aggressive Characteristics of Bladder Cancer. Clin Cancer Res 2015;21: 5391-403.

14. Hu H, Wang Y, Li Z, Zhu Y, Zhang W, Wang D, et al. Overexpression of suppressor of zest 12 is associated with cervical node metastasis and unfavorable prognosis in tongue squamous cell carcinoma. Cancer Cell Int 2017;17: 26.

15. Lee W, Teckie S, Wiesner T, Ran L, Prieto GC, Lin M, et al. PRC2 is recurrently inactivated through EED or SUZ12 loss in malignant peripheral nerve sheath tumors. Nat Genet 2014:46: 1227-32.

16. Liu YL, Gao X, Jiang Y, Zhang G, Sun ZC, Cui BB, et al. Expression and clinicopathological significance of EED, SUZ12 and EZH2 mRNA in colorectal cancer. J Cancer Res Clin Oncol 2015;141: 661-9.
17. Li H, Cai Q, Wu H, Vathipadiekal V, Dobbin ZC, Li T, et al. SUZ12 promotes human epithelial ovarian cancer by suppressing apoptosis via silencing HRK. Mol Cancer Res 2012;10: 1462-72.

18. Liu C, Shi X, Wang L, Wu Y, Jin F, Bai C, et al. SUZ12 is involved in progression of non-small cell lung cancer by promoting cell proliferation and metastasis. Tumour Biol 2014;35: 6073-82.

19. Xia R, Jin FY, Lu K, Wan L, Xie M, Xu TP, et al. SUZ12 promotes gastric cancer cell proliferation and metastasis by regulating KLF2 and E-cadherin. Tumour Biol 2015;36: 5341-51.

20. Cho YJ, Kim SH, Kim EK, Han JW, Shin K, Hu H, et al. Prognostic implications of polycomb proteins ezh2, suz12, and eed1 and histone modification by H3K27me3 in sarcoma. Bmc Cancer 2018;18.

21. Chen T, Gu C, Xue C, Yang T, Zhong Y, Liu S, et al. LncRNA-uc002mbe.2 Interacting with hnRNPA2B1 Mediates AKT Deactivation and p21 Up-Regulation Induced by Trichostatin in Liver Cancer Cells. Front Pharmacol 2017;8: 669.

22. Huang Y, Wang K, Gu C, Yu G, Zhao D, Mai W, et al. Berberine, a natural plant alkaloid, synergistically sensitizes human liver cancer cells to sorafenib. Oncol Rep 2018;40: 1525-32.

23. Qin $\mathrm{Q}, \mathrm{Xu} \mathrm{Y}, \mathrm{He} \mathrm{T}$, Qin $\mathrm{C}, \mathrm{Xu}$ J. Normal and disease-related biological functions of Twist1 and underlying molecular mechanisms. Cell Res 2012;22: 90-106.

24. Giannelli G, Koudelkova P, Dituri F, Mikulits W. Role of epithelial to mesenchymal transition in hepatocellular carcinoma. J Hepatol 2016;65: 798-808.

25. Nieto MA, Huang RY, Jackson RA, Thiery JP. EMT: 2016. Cell 2016;166: 21-45.

26. Ahmed M, Uddin S, Hussain AR, Alyan A, Jehan Z, Al-Dayel F, et al. FoxM1 and its association with matrix metalloproteinases (MMP) signaling pathway in papillary thyroid carcinoma. J Clin Endocrinol Metab 2012;97: E1-13.

27. He X, Dong X, Zou D, Yu Y, Fang Q, Zhang Q, et al. Enantioselective Effects of o,p'-DDT on Cell Invasion and Adhesion of Breast Cancer Cells: Chirality in Cancer Development. Environ Sci Technol 2015;49: 10028-37.

28. Chen L, Guo P, He Y, Chen Z, Chen L, Luo Y, et al. HCC-derived exosomes elicit HCC progression and recurrence by epithelial-mesenchymal transition through MAPK/ERK signalling pathway. Cell Death Dis 2018;9: 513.

29. Liu Y, Zhang $X$, Yang B, Zhuang $H$, Guo $H$, Wei $W$, et al. Demethylation-Induced Overexpression of Shc3 Drives c-Raf-Independent Activation of MEK/ERK in HCC. Cancer Res 2018;78: 2219-32.

30. Morris EJ, Jha S, Restaino CR, Dayananth P, Zhu H, Cooper A, et al. Discovery of a novel ERK inhibitor with activity in models of acquired resistance to BRAF and MEK inhibitors. Cancer Discov 2013;3: 742-50.

31. Andrisani O. Deregulation of Epigenetic Mechanisms by the Hepatitis B Virus $X$ Protein in Hepatocarcinogenesis. Viruses 2013;5: 858-72.

32. Peng F, Jiang J, Yu Y, Tian R, Guo X, Li X, et al. Direct targeting of SUZ12/ROCK2 by miR-200b/c inhibits cholangiocarcinoma tumourigenesis and metastasis. Brit J Cancer 2013;109: 3092-104.

33. Zhang H, Diab A, Fan H, Mani SKK, Hullinger R, Merle P, et al. PLK1 and HOTAIR Accelerate Proteasomal Degradation of SUZ12 and ZNF198 during Hepatitis B Virus - Induced Liver Carcinogenesis. Cancer Res 2015;75: 2363-74.

34. Fan $\mathrm{H}$, Zhang $\mathrm{H}$, Pascuzzi $\mathrm{PE}$, Andrisani $\mathrm{O}$. Hepatitis $\mathrm{B}$ virus $\mathrm{X}$ protein induces EpCAM expression via active DNA demethylation directed by RelA in complex with EZH2 and TET2. Oncogene 2016;35: 715-26.

35. Ma Z, Xin Z, Hu W, Jiang S, Yang Z, Yan X, et al. Forkhead box O proteins: Crucial regulators of cancer EMT. Semin Cancer Biol 2018;50: 21-31.

36. Comet I, Riising EM, Leblanc B, Helin K. Maintaining cell identity: PRC2-mediated regulation of transcription and cancer. Nat Rev Cancer 2016;16: 803-10.

37. Kidger AM, Sipthorp J, Cook SJ. ERK1/2 inhibitors: New weapons to inhibit the RAS-regulated RAF-MEK1/2-ERK1/2 pathway. Pharmacol Ther 2018;187: 45-60.

38. Guo $Y$, Peng $Y$, Gao D, Zhang $M$, Yang W, Linghu E, et al. Silencing HOXD10 by promoter region hypermethylation activates ERK signaling in hepatocellular carcinoma. Clin Epigenetics 2017;9: 116.

39. Wang $\mathrm{K}, \mathrm{Ji} \mathrm{W}, \mathrm{Yu}$ Y, Li Z, Niu X, Xia W, et al. FGFR1-ERK1/2-SOX2 axis promotes cell proliferation, epithelial-mesenchymal transition, and metastasis in FGFR1-amplified lung cancer. Oncogene 2018.

40. Baker A, Wyatt D, Bocchetta M, Li J, Filipovic A, Green A, et al. Notch-1-PTEN-ERK1/2 signaling axis promotes HER2+ breast cancer cell proliferation and stem cell survival. Oncogene 2018. 